\title{
The Research on Tetrahedral Mesh Optimization Algorithm of the Double Channel Pump
}

\author{
Liu Houlin ${ }^{1}$, Lu Mingzhen ${ }^{2, *}$, Tan Minggao ${ }^{1}$, Dong Liang $^{1}$ and Ren Yun ${ }^{1}$ \\ ${ }^{1}$ Research Center of Fluid Machinery Engineering and Technology, Jiangsu University, China \\ ${ }^{2}$ Department of Mechanical Engineering, The Hong Kong Polytechnic University, Hong Kong
}

Received: 15 May. 2013, Revised: 19 Sep. 2013, Accepted: 20 Sep. 2013

Published online: 1 May. 2014

\begin{abstract}
The quality of mesh generation is very important for the computational simulation of the double channel pump. According to the special characteristics of the double channel pump, there exist a great amount of poor-quality elements, most of which are so called Sliver elements, generated by the tetrahedral mesh generation algorithms of Advancing Front Technique (AFT) and Delaunay Triangulation. By studying the type, distribution and reason of the badly shaped elements, a method to eliminate these Sliver elements was proposed. Then this method was combined with advanced optimization-based smoothing, Laplacian smoothing and swapping optimization to optimize the tetrahedral mesh. The computer language $\mathrm{C}++$ is applied to develop the algorithms. Computational experiments of a double channel pump shown that the worst elements can be removed and the whole quality can be improved noticeably. After comparing the result of numerical simulation with that of the experiment, it is believed that the tetrahedral mesh optimization algorithm can satisfy the computational simulation of the double channel pump.
\end{abstract}

Keywords: Double Channel Pump, Tetrahedral Mesh Optimization, Sliver Element

\section{Introduction}

With the fast development of computer technology, Computational Fluids Dynamics (CFD) has already been used in engineering widely. Mesh generation technology, one of the key CFD techniques, affects the simulation precision of numerical calculation directly. At present, the common methods of fully automatic 3D finite element mesh generation are Advancing Front Technique (AFT), Delaunay triangulation and Quad-tree/Octree, etc. [1,2,3, 4] However these algorithms can inevitably generate the badly shaped elements, which will reduce the accuracy of numerical calculation, even disrupt the calculation. Generally speaking there are much more badly shaped elements in $3 \mathrm{D}$ tetrahedral mesh than those in $2 \mathrm{D}$ elements. Therefore, the 3D tetrahedral mesh optimization becomes another key point after the mesh automatic generation technology.

Different modeling methods of pumps result in different requirements for mesh generation. In order to improve the model accuracy, a great amount of contour lines may be included in the double channel model. The "smash surfaces" was caused by these excessive contour lines, especially by the excessive contour lines which are too close. For the commercial mesh generator Gambit, these smash surfaces need to be merged and other complicated operations before mesh can be generated successfully.

However, this step is no needed for full automatic mesh generation algorithms before the mesh generation. It is easy for these algorithms to generate the mesh whatever the pump models were. However, many badly-shaped elements can be generated because of the limitations of the algorithms and the contour lines. Therefore it is necessary to optimize the mesh in the pump model after the mesh generation.

\section{The Type of Badly Shaped Elements}

The badly shaped elements of tetrahedral mesh can be classified the following three types [5]:

Type I: one edge is too short as compared with the others.

Type II: one vertex is close to the centroid of the two or three others.

\footnotetext{
*Corresponding author e-mail: mingzhen.lu@ connect.polyu.edu.hk
} 


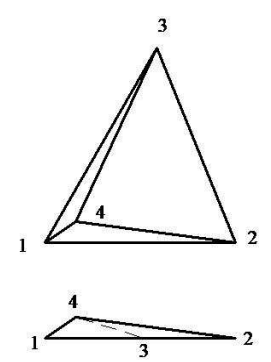

Type I
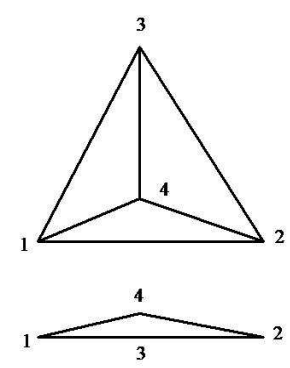

Type II
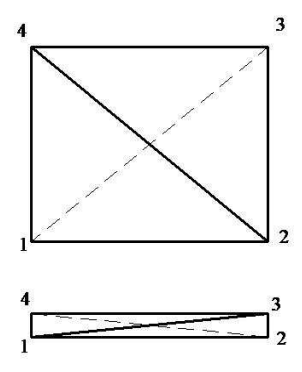

Type Ш

Fig. 1: The three types of badly shaped elements

Type III: the four vertices almost form a planar square (Sliver elements).

Meanwhile, the type elements can be classified into three situations according to the different positions of the peak point projection: the projection in or out of the triangle, the project point at the edge of the triangle(Fig. 1). Also, the right hand rule should be satisfied for the three types of tetrahedral element.

\section{The Numerical Estimate Criteria of Badly Shaped Elements}

There are four triangles for the three badly shaped elements. Make the area of triangle 123 be the maximum area, centroid $0\left(X_{0}, Y_{0}\right)$ of the triangle 123 can be got when point 4 project to triangle 123 . The coordinate of 0 are as following:

$$
X_{0}=\frac{X_{1}+X_{2}+X_{3}}{3}, Y_{0}=\frac{Y_{1}+Y_{2}+Y_{3}}{3}
$$

The area of triangle 123 is $S_{123}=S_{\max }=\max \left(S_{123}, S_{023}, S_{012}, S_{013}\right)$. The distance between point 0 and 4 is:

$$
D=\sqrt{\left(\mathrm{X}_{0}-\mathrm{X}_{4}\right)^{2}+\left(\mathrm{Y}_{0}-\mathrm{Y}_{4}\right)^{2}}
$$

Make A equals to $1.0 \times 10^{-5}$, nearly equals to zero. Then the numerical estimation criteria of the badly shaped elements are as following: If $S_{123}=S_{012}+S_{023}+S_{013}$, the type of the badly shaped elements may be type I or type II; Meanwhile, if $D<A$, the badly shaped elements must be type II Otherwise, the badly shaped elements must be type I If $S_{123} S_{012}+S_{023}+S_{013}$ and the quality of the elements $Q<A$, the badly shaped elements must be type III.

\section{The Type and Distribution of Badly Shaped Elements in The Double Channel Pump}

Fig. 2 shows the distribution of badly shaped elements in one double channel pump. The tetrahedral meshes are generated by combing the AFT with Delaunay triangulation.

As shown in Fig. 2, although the model of this double channel pump was simplified to reduce the contour lines. There are still badly shaped elements in the double channel pump because of the high distortion and the regions containing contour lines. Owing to the decrease of contour lines, the badly shaped elements are mostly generated by the mesh generation algorithms and the high distortion of the model. The types of badly shaped elements are type I and type III. The most badly shaped element is generated in the volute whose quality $\eta[6,7$, $8]$ is near to zero. All of those badly shaped elements generated in the double channel pump should be eliminated.

\section{The Mesh Optimization Algorithms}

\subsection{The "Smart” Laplacian Smoothing}

The most common smoothing technique is Laplacian smoothing. According to the mesh quality measurement, "Smart" Laplacian smoothing technique relocates each of the mesh node to the geometric center of adjacent grid points only if the quality of the local mesh could be improved. This technique generally quite suits for meshes in convex regions. However, the single Laplacian smoothing could result in inverted elements near concavities in the model, it is always used combining other mesh optimization algorithms $[9,10,11]$.

\subsection{The Optimization-based Smoothing}

The Optimization-based smoothing $[12,13]$ that uses function and gradient evaluations find the minimum (or maximum) value of the composite function (8).

$$
\phi(x)=\min _{1 \leq i \leq n} f_{i}(x)
$$


(a)

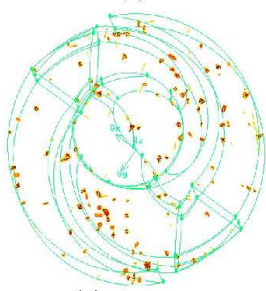

(c)

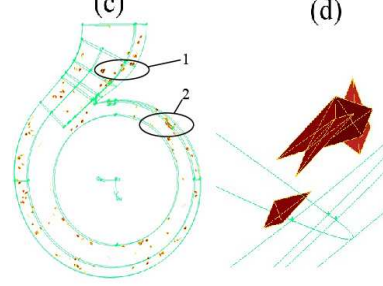

(f) (b)

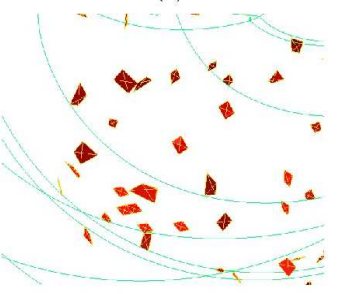

(e)

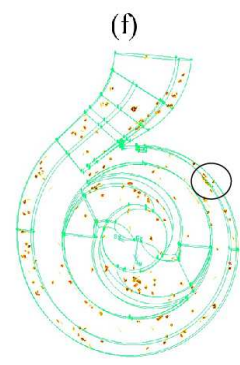

(g)

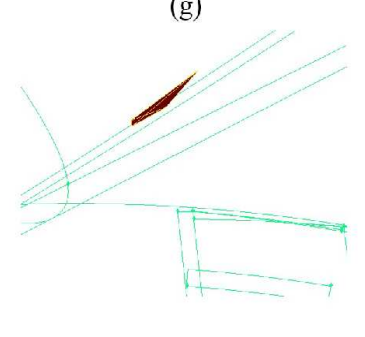

Fig. 2: The Badly shaped elements distribution in the impeller, volute and the whole pump is shown in (a), (c) and (f), respectively. The (b), (d), (e) and (g) are the zoom window of the elements. It is indicated that the sliver element is the most elements in the double channel pump

where $f_{i}(x)$ is the quality measure function of the elements, $\varphi(x)$ is the composite function. For different distortion metric, the functions $f_{i}(x)$ are different. The selection of a good distortion metric is very important. Many a priori metrics have been developed $[14,15,16$, 17]. However, no optimized objective function was proposed.

To obtain an optimized objective function, an error function was proposed. For a tetrahedral element T, the quality of $\mathrm{T}$ is as below [5].

$$
Q_{e}(T)=\alpha \frac{r}{h}
$$

where $h$ is the longest edge, $r$ is the in-radius and is the scaling factor to make the quality of an equilateral element is 1 . Thus, the quality varies in the interval $[0,1]$, from the worst quality (close to 0 ) to the best quality equals to 1 .

The quality of an edge $\mathrm{AB}$, is defined as follow [5]:

$$
Q_{h}(A B)=\min \left(\frac{l}{h}, \frac{h}{l}\right)
$$

where 1 is the length of $A B$. The quality varies in the interval $[0,1]$ as well.

According to the in-radius formula:

$$
r=\frac{3 V}{S_{1}+S_{2}+S_{2}+S_{3}}
$$

where $S_{1}, S_{2}, S_{3}, S_{4}$ are the areas of the four surfaces of the element respectively, $\mathrm{V}$ is the volume.

The error function of one element can be derived:

$$
E(T)=\frac{1}{6 \sqrt{6}} \frac{\left(\sum_{i=1}^{4} A\left(f_{i}\right)\right) \max _{i=1, . .6} l_{i}}{V}+\sum_{i=1}^{6}\left(\frac{l_{i}}{h}-\frac{h}{l_{i}}\right)^{2}
$$

Thus, when the element is an equilateral element, the value of the first term is 1 and the second term is 0 to make sure the error of an equilateral element is 1 . As one element degenerates, the function tends to infinite. The total error function of the mesh is the sum of all elements errors.

$$
E(\Gamma)=\sum_{T \in \Gamma} E(T)
$$

Theoretically, the less value of the error function the better quality of the mesh. So, the variable metric method is adopted to solve the minimum of the error function that can be optimized the quality of the tetrahedral mesh. 


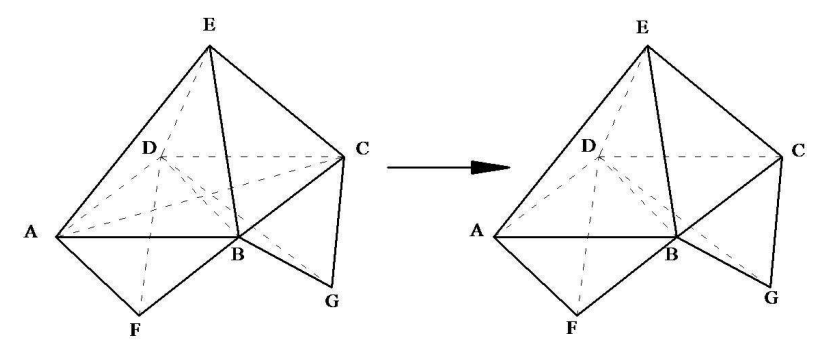

Fig. 3: The first elimination method of sliver element

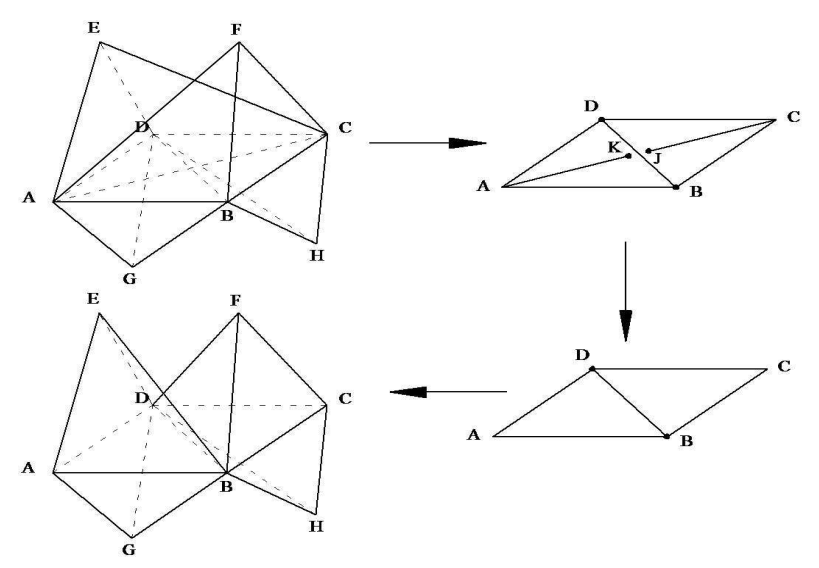

Fig. 4: The second elimination method of sliver element

\subsection{The Elimination Method of Sliver Element}

As analyzed above, the most type of badly shaped elements in the double channel pump is type III (sliver element). The sliver element is mostly generated by Delaunay triangulation algorithm. Those sliver elements can cause inaccurate result for the numerical calculation. Because of its special shape, it is hard to be eliminated by the normal mesh optimization algorithms, such as swapping and smoothing. Therefore the eliminate methods for different sliver elements are proposed.

There are two types of sliver element proposed by Canvendish [18]. The first type is the two conjoined tetrahedrons that share one vertex.

As shown in Fig. 3, the tetrahedral element ABCD is sliver element connected with the element of $\mathrm{ABCE}$, ACDE, ADBF and BDCG. The elements of ABCE and ACDE share one vertex E. It just needs to delete the ABCD from the map of elements to eliminate this sliver element. By transforming the elements of $\mathrm{ABCE}$ and $\mathrm{ACDE}$ into $\mathrm{ABDE}$ and $\mathrm{BCDE}$ the silver elements can be eliminated relatively easily.

The second type is opposite to the first type that the two conjoined tetrahedrons do not share one vertex.

As shown in Fig. 4 that the tetrahedral element ABCD is sliver element connected with the elements of $\mathrm{ABCF}$,
ACDE, ADBG and BDCH. None of any two of the tetrahedral elements shares one vertex. In this type of sliver elements, the sliver element cannot be removed by swapping and smoothing method. The elimination for this sliver element was proposed using point insertion and collapsing. Firstly, the points $\mathrm{K}$ and $\mathrm{J}$ are inserted into the maximum edge AC. The edge $\mathrm{AC}$ can be divided into edge AK and JC. Then the point collapsing was used to superpose point $\mathrm{K}$ and $\mathrm{J}$ with $\mathrm{B}$ and $\mathrm{D}$. The point $\mathrm{K}$ and $\mathrm{J}$ cannot be superposed the points which belong to. This means that point $\mathrm{K}$ and $\mathrm{J}$ cannot overlap point $\mathrm{A}$ and $\mathrm{C}$. In the end, the tetrahedral elements ABCF and ACDE need to be deleted from the map. Then the original tetrahedral elements map can be transformed into ABDE, BCDF, $\mathrm{ADBG}$ and $\mathrm{BDCH}$. As a result the sliver elements can be removed as well.

In addition to these two types of silver element, if one edge of the sliver element belongs to the boundary, it is also cannot be removed by swapping. However, the silver element can also be removed by the second elimination method. 


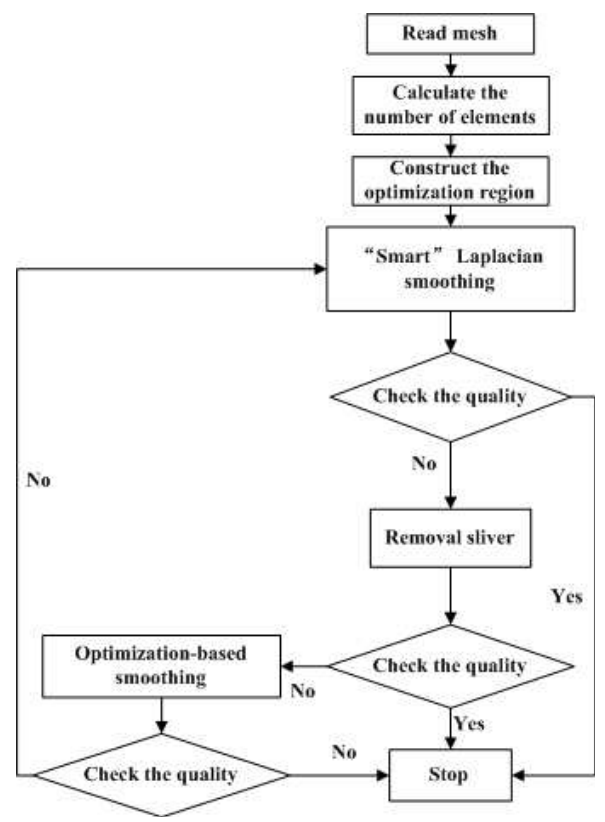

Fig. 5: Program flow process

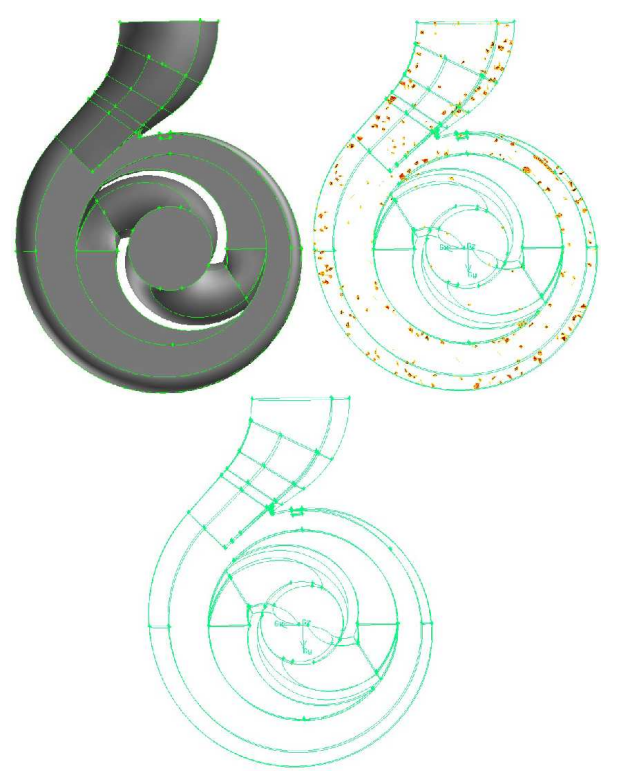

Fig. 6: The distribution of badly shaped elements before and after optimization

\section{The Combination of Mesh Optimization Algorithms}

There are many limitations for each of the mesh optimization algorithm above. So the three mesh optimization algorithms are combined to optimize the tetrahedral mesh of double channel pump. The program flow process is stated in Fig. 5.

\section{Computational Experiments}

The application program is developed by $\mathrm{C}++$. The operation system is Windows XP. The CPU is $2.0 \mathrm{~Hz}$ and the memory is $1.0 \mathrm{G}$. The tetrahedral mesh is generated by the open source code Gmsh [19]. The design parameters and experiment performance of the double channel pump are shown in table 1 . The tetrahedral mesh consists of 


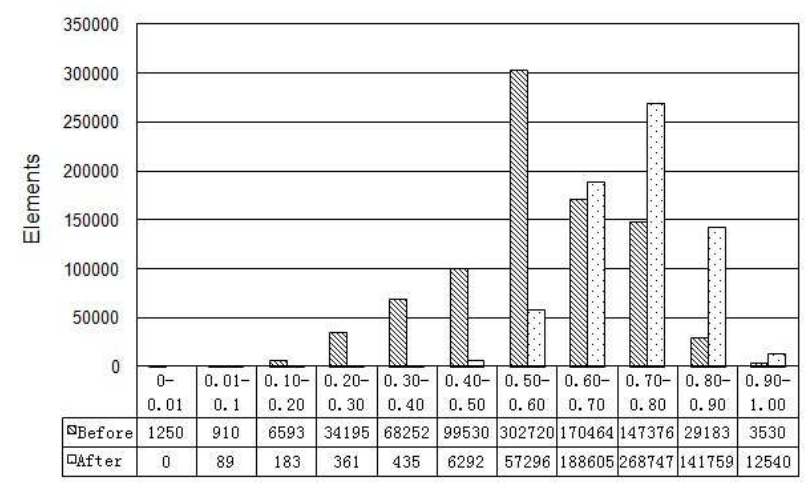

Fig. 7: Mesh quality before and after optimization

Table 1: The design parameters and test performance

\begin{tabular}{cccc}
\hline Flow rate & $Q$ & 55 & $\mathrm{~m}^{3} \cdot \mathrm{h}^{-1}$ \\
Head & $H$ & 23.49 & $\mathrm{~m}$ \\
Rotation speed & $n$ & 2900 & $\mathrm{r} \cdot \mathrm{min}^{-1}$ \\
Specific speed & $n_{s}$ & 129 & \\
Efficiency & $\eta$ & 60.77 & $\%$ \\
Number of blade & $Z$ & 2 & \\
Impeller outlet diameter & $D_{2}$ & 155 & $\mathrm{~mm}$ \\
Impeller outlet width & $b_{2}$ & 38 & $\mathrm{~mm}$ \\
Volute base circle diameter & $D_{3}$ & 180 & $\mathrm{~mm}$ \\
Volute inlet width & $b_{3}$ & 48 & $\mathrm{~mm}$ \\
Volute outlet diameter & $D_{4}$ & 80 & $\mathrm{~mm}$ \\
\hline
\end{tabular}

168754 nodes and 1003706 elements. The $n_{s}$ is $3.65 n(Q)^{0.5} / H^{0.75}$.

Fig. 6 indicates that before optimization, there are quantities of badly shaped elements. While after optimization, the badly shaped elements nearly all removed.

As shown in Fig. 7 that, there are a great amount of sliver elements whose qualities are less than 0.01. Then after optimization, the elements whose quality are less than 0.01 are all removed and the whole mesh qualities are better than the original mesh. The qualities of most badly shaped elements are around $3.3810^{-5}$. After being optimized most of them are increased around to 0.05 .

\section{Conclusion}

The 3D tetrahedral mesh generation algorithms and the contour lines of the double channel pumps may produce many badly shaped elements in the model of double channel pump. These badly shaped elements can cause the decrease of the numerical calculation accuracy or even stop the calculation.

The types of badly shaped elements generated in the double channel pump are type and type. The advanced optimization-based smoothing was proposed using the error function. The type elements are sliver elements. Different elimination methods of sliver elements were proposed as well.

The mesh optimization for double channel pump combining "Smart" Laplacian smoothing, advanced optimization-based smoothing with sliver elements elimination methods is used. Compared with the qualities before and after optimization it can be concluded that the mesh optimization algorithms above can eliminate the badly shaped elements in the double channel pump efficiency. The advanced optimization-based smoothing and sliver elements elimination methods are useful for the optimization of tetrahedral elements in the double channel pump.

\section{Acknowledgement}

This work was supported by grand 51079062 from the National Natural Science Foundation of China and grand BK2010346 from Natural Science Foundation of Jiangsu Province, China.

The authors are grateful to the anonymous referee for a careful checking of the details and for helpful comments that improved this paper. 


\section{References}

[1] R. A Lohner, Proceedings of 38th AIAA Aerospace Science Meeting, 51, 10-13 (2000).

[2] D. Watson, The Computer Journal, 24, 167-172 (1981).

[3] P. J. Frey and M. Loic, Proceedings of the 7th International Meshing Roundtable, Dearborn, 211-224 (1998).

[4] Rebay, Journal of Computational Physics, 106-125 (1993).

[5] I. Babuska, J.E. Flaherty and W.D. Henshaw, etc al., Modeling, mesh generation, and adaptive numerical methods for partial differential equations, 97-127 (1995).

[6] A. Liu and B. Joe, Relationship between tetrahedron shape measures, BIT, 34, 268-287 (1994).

[7] C. G. Nie, J. F. Liu and S. L. Sun, Chinese Journal of Computational Mechanics, 20, 579-582 (2003).

[8] Z. Q. Guan, B. Z. Liu, Y. X. Gu , et al., Chinese Journal of Computational Mechanics, 24, 257-263 (2007).

[9] A. F. David, Communications and Applied Numerical Methods, 4, 709-712 (1988).

[10] J. R. Schewchul, Computing and Visualization in Science, 1, 41-52 (1997).

[11] S. H. Lo, International Journal for Numerical Methods in Engineering, 21, 1403-1426 (1985).

[12] L. A. Freitag and C.O. Gooch, Int J for Numerical Methods in Engineering, 40, 3979-4002 (1997).

[13] L. A. Freitag, AMD Trend in Unstructured Mesh Generation, ASME., 220, 37-43 (1997).

[14] R. E. Bank and R. K. Smith, SIAM Journal on Numerical Analysis, 34, 979-997 (1997).

[15] J. K. Riccius, Schweizerhof, M. Baumann, International Journal for Numerical Methods in Engineering, 40, 24592474 (1997).

[16] O. P. Jacquotte, G. Coussement, Computer Methods in Applied Mechanics and Engineering, 101, 397-432 (1992).

[17] J. M. Escobar, R. Montenegro, G. Montero, et al., Computers and Structures, 83, 2423-2430 (2005).

[18] J. C. Canvendish, D. A. Field and W. H. Frey, Int. J. Num. Methods Eng., 21, 329-347 (1985).

[19] C. Geuzaine, J. F. Remacle, Int. J. Num. Methods Eng., 0, 1-24 (2009).

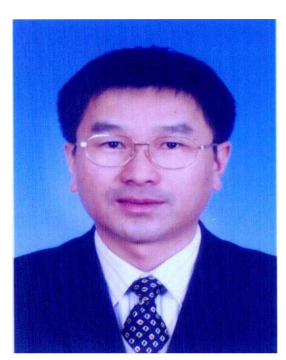

Liu Houlin received the $\mathrm{PhD}$ in Fluid Machinery Engineering from Jiangsu University. He is currently a Professor with Research Center of Fluid Machinery Engineering and Technology at Jiangsu University, China. He has published more than 80 papers. His research interests are in the areas of the theory, design, CAD and CFD of pumps.

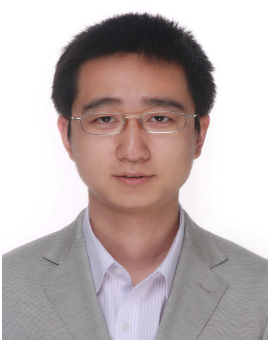

Lu Mingzhen received his MS degree in Fluid Machinery Engineering from Jiangsu University. $\mathrm{He}$ is currently a $\mathrm{PhD}$ Student with the Department of Mechanical Engineering at The Hong Kong Polytechnic University. His research interests are in the areas of the mesh quality metric, mesh quality improvement and CFD simulation.

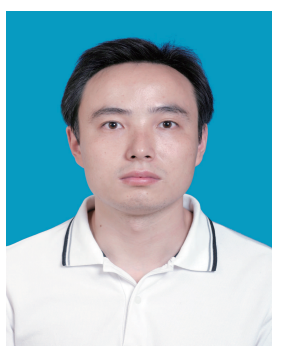

Tan Minggao received the $\mathrm{PhD}$ in Fluid Machinery Engineering from Jiangsu University. $\mathrm{He}$ is currently an assistant research fellow with Research Center of Fluid Machinery Engineering and Technology at Jiangsu University. He has published more than 20 papers. His research interests are in the areas of CFD, design and performance prediction of pumps.

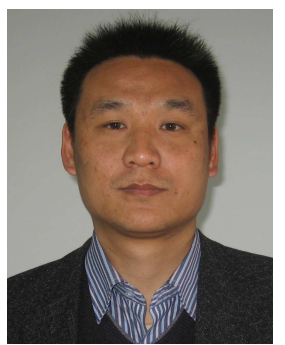

Dong Liang received the MS degree in Fluid Machinery Engineering from Jiangsu University. $\mathrm{He}$ is currently a $\mathrm{PhD}$ Student in Research Center of Fluid Machinery Engineering and Technology. His research interests are in the areas of mesh generation, mesh quality metric and mesh

quality improvement.

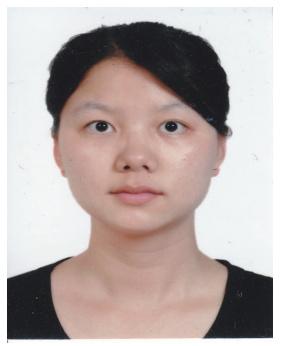

Ren Yun received the MS degree in fluid machinery engineering from Jiangsu University. She is currently an $\mathrm{PhD}$ student in Research Center of Fluid Machinery Engineering and Technology. Her research interests are in the areas of numerical simulation of pump, SIMPLE algorithm understanding,

turbulence model improvement and LES. 